\title{
Cancer cells escape p53's tumor suppression through ablation of ZDHHC1-mediated p53 palmitoylation
}

Jun Tang ${ }^{1,7}$, Weiyan Peng ${ }^{1,7}$, Yixiao Feng ${ }^{1,7}$, Xin Le ${ }^{1}$, Kang Wang ${ }^{1}{ }^{1}$, Qin Xiang ${ }^{1}$, Lili Li $i^{2}$, Yan Wang ${ }^{1}$, Can Xu ${ }^{1}$, Junhao Mu ${ }^{1}$, Ke Xu ${ }^{1}$, Ping $\mathrm{Ji}^{3}$, Qian Tao $\mathbb{D}^{1,2}$, Ailong Huang ${ }^{4}$, Chu-Xia Deng $\mathbb{D}^{5}$, Yong Lin $^{6}$ and Tingxiu Xiang $\mathbb{D}^{1 凶}$

(c) The Author(s) 2021

The inactivation of tumor-suppressor genes contributes heavily to oncogenesis. The mutation of TP53 has been well-studied and recognized as a major factor in the development of tumors. Yet other means of p53 inactivation has not been well-elucidated. We previously identified a hypermethylated gene ZDHHC1 that suppresses tumor growth when the expression was restored, but the specific mechanism was yet to be found. The protein product of ZDHHC1 is an S-palmitoyltransferase and we have identified p53 as a substrate for ZDHHC1-mediated palmitoylation, specifically at the C135, C176, and C275 residues. The novel form of posttranslational modification of p53 is required for the nuclear translocation of the tumor suppressor. p53 recruited DNMT3A to ZDHHC1 promoter and is responsible for the hypermethylation of ZDHHC1. The epigenetic feedback loop formed by ZDHHC1 and p53 sheds light on the inactivation of p53 without the presence of genetic mutations.

Oncogene (2021) 40:5416-5426; https://doi.org/10.1038/s41388-021-01949-5

\section{INTRODUCTION}

Cancer development is a complex process involving oncogene activation and tumor suppressor gene inactivation by genetic and/or epigenetic mechanisms [1]. TP53 is one of the most extensively studied tumor suppressor genes whose multifaceted mechanisms involve apoptosis, ferroptosis, DNA repair, genomic stabilization, and angiogenesis [2]. Thus, breaking through p53's blockade is a crucial step for malignant cellular transformation. Approximately half of the human solid tumors have one or more mutations in the genetic sequence of TP53, which often leads to the loss of tumor-suppressing properties and even worse, the gain of tumor-promoting functions [3]. However, in cancers retaining wild-type TP53 (TP53 $\left.{ }^{\mathrm{WT}}\right)$, the mechanism of tumorigenesis is not well-understood.

Beyond the genetic level, p53 activity is mainly regulated by post-translational modifications (PTMs). For example, p53 protein is activated by phosphorylation and acetylation and inactivated by ubiquitination-mediated proteasomal degradation [4]. When triggers such as DNA damage is induced, p53 is rapidly phosphorylated, which triggers its nuclear translocation to regulate the expression level of its target genes. The transcription regulating the activity of $\mathrm{p} 53$, either activation or repression, is further modulated by $\mathrm{p} 300$-mediated acetylation on the promoter [5]. However, the current understanding of p53 PTMs fails to translate into effective prevention or treatment of cancers and requires further exploration.

Protein S-palmitoylation is recently defined as another important form of post-translational modification in tumorigenesis [6, 7], which is catalyzed by polytopic transmembrane proteins named protein acyltransferases (PATs) with zinc-finger and aspartate-histidinehistidine-cysteine (DHHC) motifs $[8,9]$. Multiple groups showed that members of the zDHHC family can both inhibit [10] and promote tumorigenesis by modulating S-palmitoylation of crucial proteins such as EGFR [11] and PD-L1 [12].

In our earlier work, we have identified Zinc Finger DHHC-Type Containing 1 (ZDHHC1) as a potential tumor suppressor that is hypermethylated in many cancers [13]. We further investigated the features of ZDHHC1 and found that ZDHHC1 suppressed tumor cell growth by activating p53 on many levels, most importantly by mediating p53 S-palmitoylation. Interestingly, p53 in turn induced $Z D H H C 1$ hypermethylation by recruiting DNMT3A to the p53 binding motif in the $Z D H H C 1$ promoter. These findings suggest a novel form of PTM for p53 that is critical in maintaining the activation of the p53 pathway, which is a part of an epigenetic regulatory loop between $\mathrm{ZDHHC} 1$ and $\mathrm{p} 53$, thus shedding light on the regulation of $\mathrm{p} 53$ pathway without the occurrence of genetic mutations and providing potential therapeutic targets for the battle against cancer.

\section{RESULTS}

ZDHHC1 is suppressed in TP53 ${ }^{W T}$ cancers and ectopic ZDHHC1 inhibits the proliferation of TP53 ${ }^{W T}$ cancer cells

Our previous work suggested that ZDHHC1 was silenced or downregulated in many cancer cell lines and tissues due to

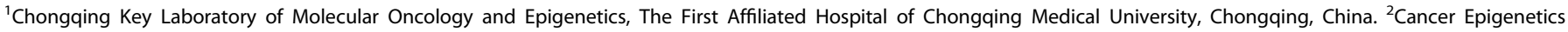

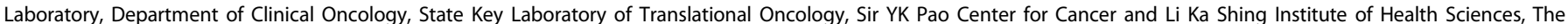

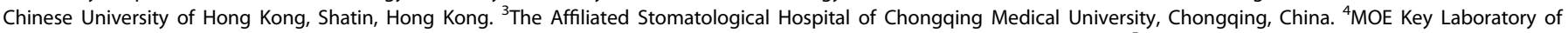

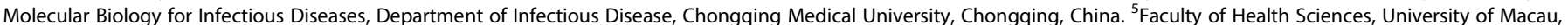
Macau, SAR, China. ${ }^{6}$ Lovelace Respiratory Research Institute, Albuquerque, NM, USA. ${ }^{7}$ These authors contributed equally: Jun Tang, Weiyan Peng, Yixiao Feng.

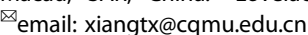


A

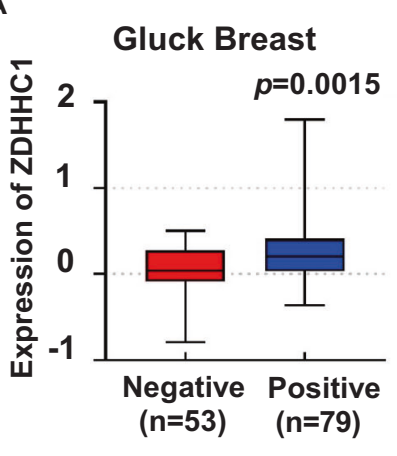

D
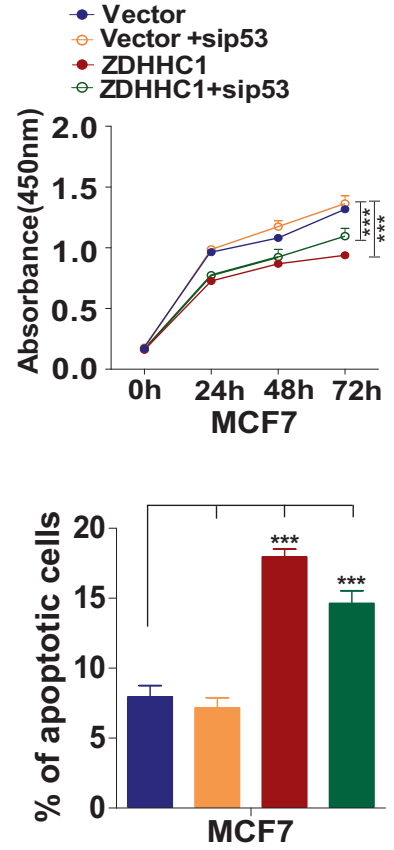

B

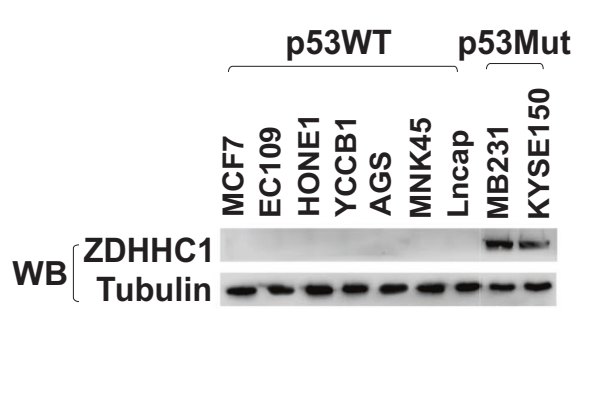

C
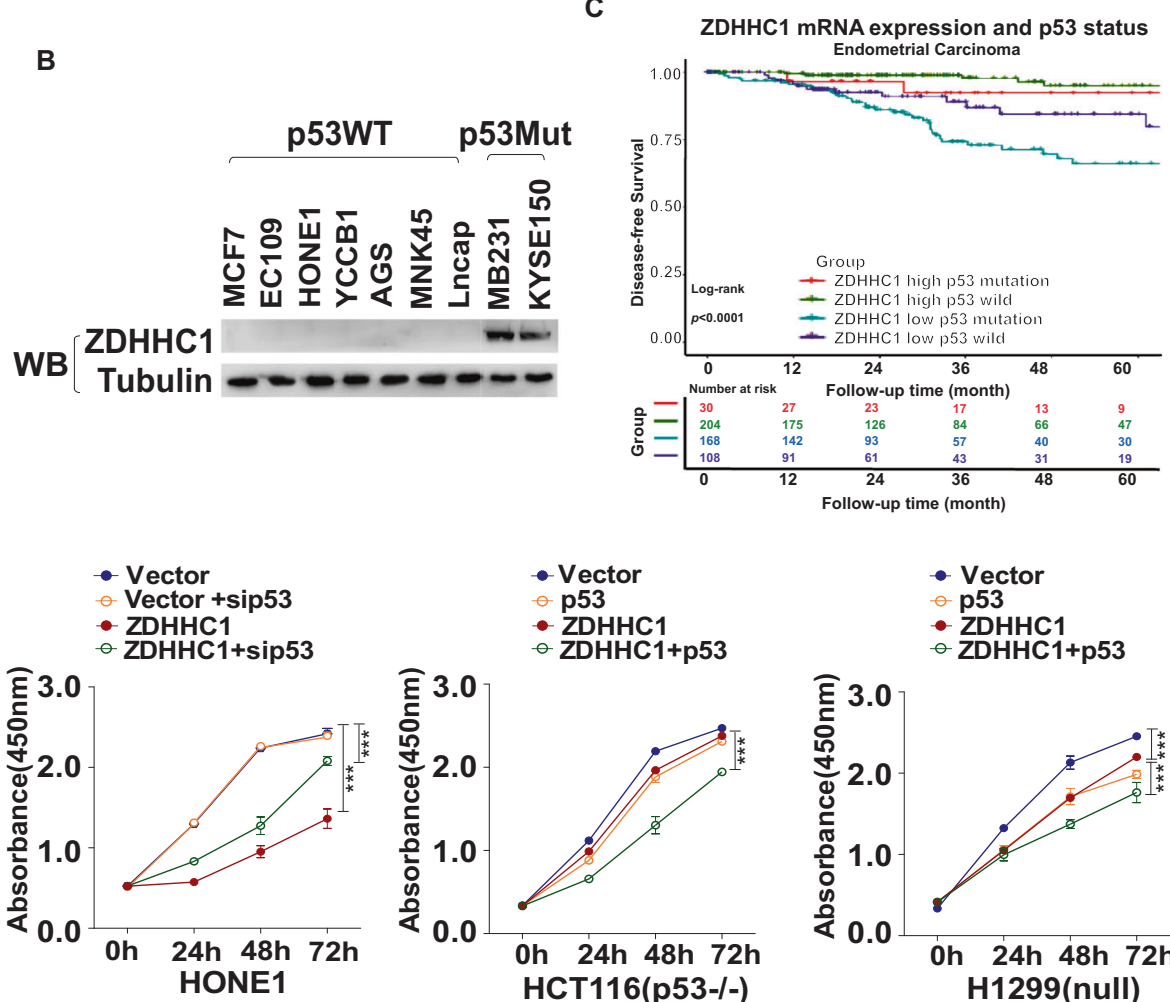
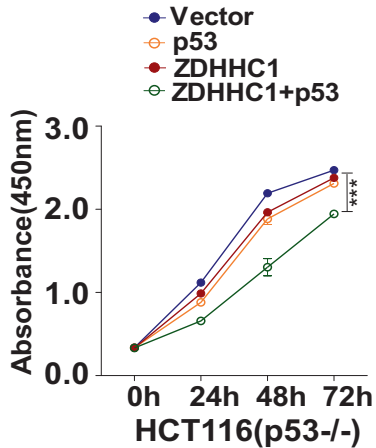

HCT116(p53-/-)

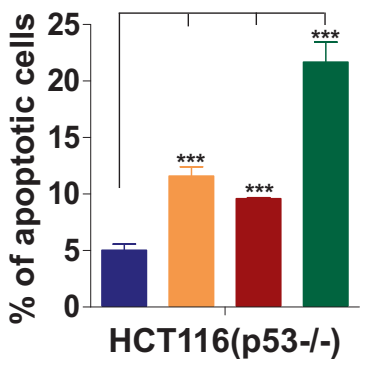

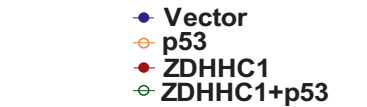
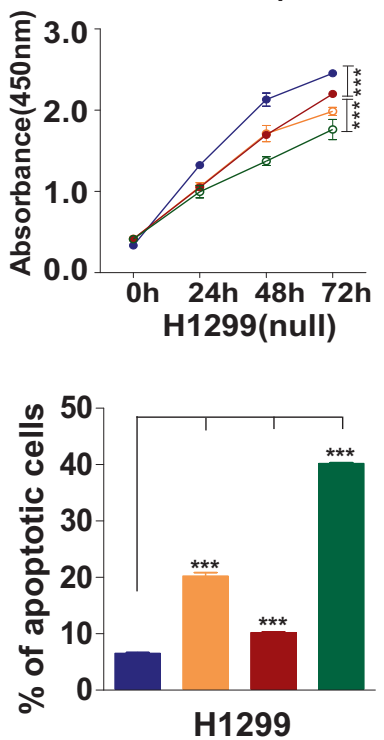
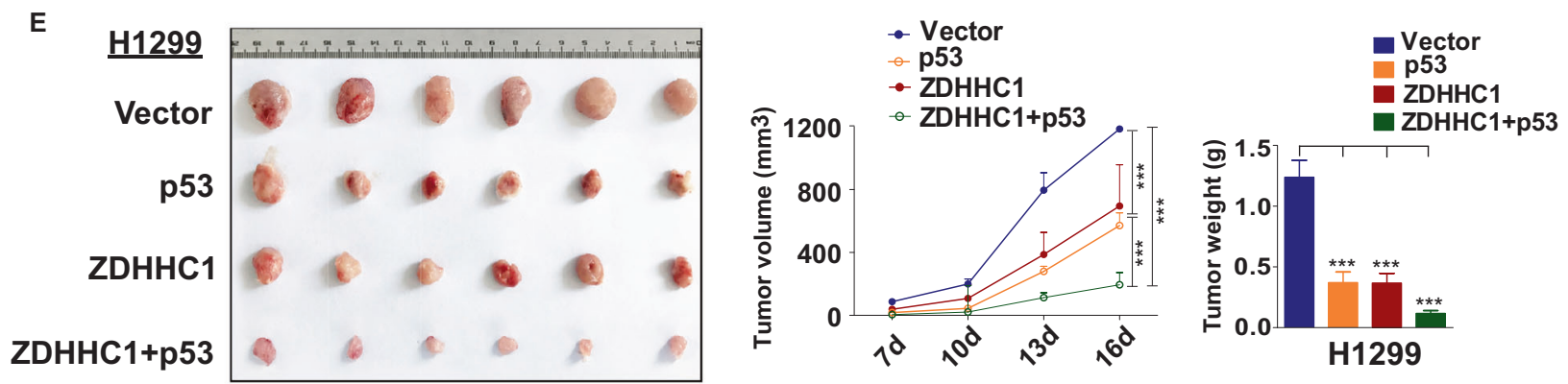

Fig. 1 ZDHHC1 expression and tumor-suppressing function correlate with p53 status. A ZDHHC1 mRNA expression in breast cancer patients [14] grouped by p53 IHC results. B ZDHHC1 protein expression in human cancer cell lines grouped by p53 mutation. C Disease-free survival of endometrial carcinoma patients according to ZDHHC1 mRNA expression and p53 mutation status. See also Fig. S1B. D CCK8 and flow cytometric analyses of human cancer cell lines' proliferation and apoptosis respectively. See also Fig. S2A-D. E Volume and weight of H1299 xenografts. See also Fig. S3A-B. Values represent mean \pm SEM. ${ }^{* * *} p<0.001$.

promoter DNA hypermethylation [13]. We've also noticed a correlation between ZDHHC1 expression and TP53 mutation. To corroborate the notion, we first established that ZDHHC1 expression was quite ubiquitous in normal human tissues (Fig. S1A). In a cohort of 132 breast cancer samples [14], ZDHHC1 mRNA expression is positively correlated with p53 immunohistochemistry staining positivity, which is considered a sign of TP53 mutation (Fig. 1A). Western blot showed ZDHHC1 expression patterns in human cancer cell lines being consistent with earlier RT-PCR results [13], which is mostly silent in TP53 ${ }^{W T}$ cells but not TP53 $3^{\text {Mut }}$ cells (Fig. 1B). Disease-free survival favors the groups of carcinoma patients with wild-type p53 and high ZDHHC1 expression while the group with mutated p53 and lower ZDHHC1 had the shortest disease-free survival (Fig. 1C; Fig. S1B). On the 
A

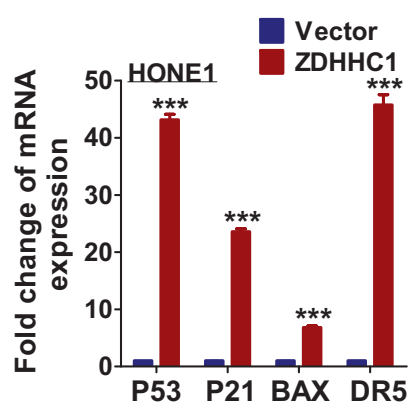

B

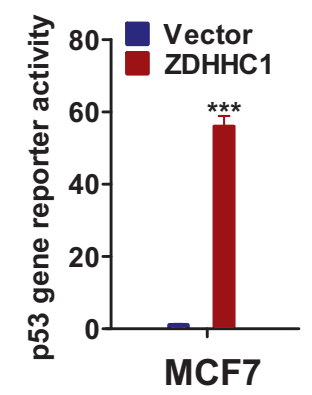

C

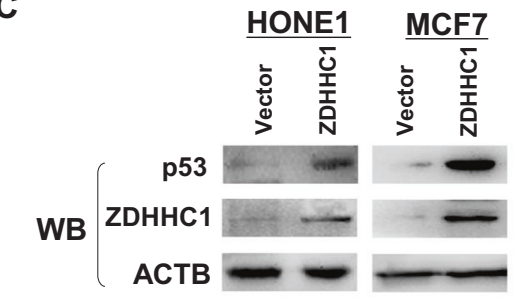

E

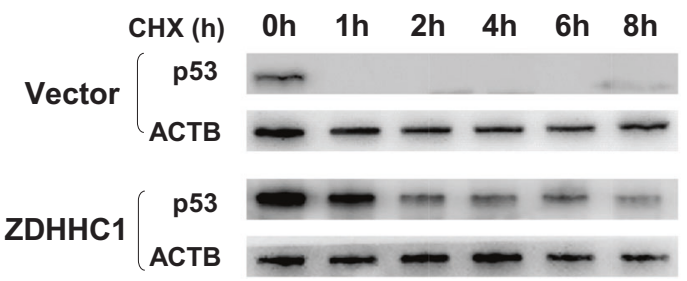

D

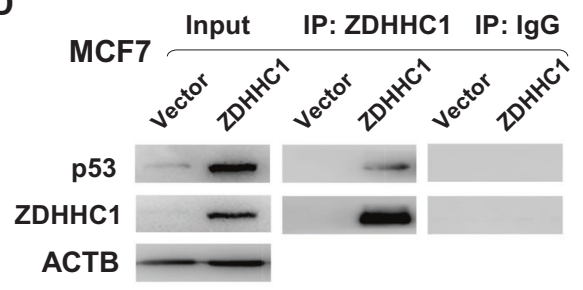

$\mathbf{F}$

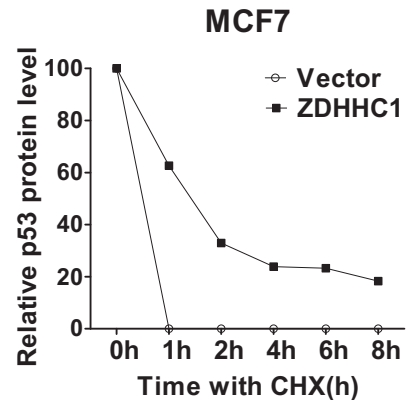

Fig. 2 ZDHHC1 activates p53 signaling through transcriptional and post-translational regulations. A mRNA expression of p53 target genes by qRT-PCR. B Transcriptional activity of TP53 promoter in MCF7 cells. C Protein expression of p53 and ZDHHC1 by Western blot. D Co-IP analysis of ZDHHC1-p53 interaction in MCF7 cells. E, F p53 degradation rate analysis by Western blot and cycloheximide (CHX) treatment in MCF7 cells. Values represent mean \pm SEM. ${ }^{* * *} p<0.001$.

cellular level, ZDHHC1 inhibited tumor cell growth by inducing apoptosis when ectopically expressed in multiple human cancer cell lines [13]. However, this anti-tumor effect was only observed in cell lines without p53 mutations, which included HONE1 (nasopharynx) and MCF7 (breast). Knockdown of p53 in these cells effectively attenuated the effects of ZDHHC1 (Fig. 1D), suggesting ZDHHC1's anti-tumor functions is noticeably dependent on $\mathrm{p} 53^{\mathrm{WT}}$. This notion was further substantiated by the in vitro growth assay of HCT116 $6^{\text {TP53-\% }}$ and H1299 (ZDHHC1-positive, TP53 ${ }^{\text {null }}$ ) cells. Overexpression of ZDHHC1 or p53 had a limited impact on the proliferation and apoptosis of the cells, but when ZDHHC1 and p53 were overexpressed simultaneously, a synergistic inhibitory effect can be seen (Fig. 1D; Fig. S2A-D). In a nude mouse xenograft tumor model, the synergy was confirmed in vivo as both p53 and ZDHHC1 impaired the growth of H1299 xenografts but the effect was significantly stronger when both were ectopically expressed (Fig. 1E; Fig. S3A; Fig. S3B). Collectively, these results suggest that $Z \mathrm{DHHC1}$ is a p53-dependent tumor suppressor, and yet $\mathrm{ZDHHC1}$ expression is suppressed in most p53 ${ }^{\mathrm{WT}}$ cancer cells.

ZDHHC1 interacts with p53 and activates p53 signaling by promoting transcription and stabilizing $\mathbf{p 5 3}$ protein

To understand the connection between $Z D H H C 1$ and TP53, we first focused on the effect of ZDHHC1 on the p53 signaling pathway. When ZDHHC1 was ectopically expressed in HONE1 and MCF7 cells, classic targets of $P 53$ such as $P 21, B A X$, and DR5 were transcriptionally activated, along with p53 itself (Fig. 2A). ZDHHC1 has almost no effect in $\mathrm{H} 1299$ and very little effect in MB231 $\left(\right.$ TP53 ${ }^{\text {mut }}$ ) on p53 target gene expression (Fig. S3C). The increased transcription of TP53 was further confirmed by luciferase reporter assay in MCF7 (Fig. 2B). On the protein level, p53 expression also increased due to ectopic ZDHHC1 (Fig. 2C). Both ectopic and endogenous $\mathrm{ZDHHC} 1$ protein interacted with p53 protein in MCF7 and HCT116 cells respectively (Fig. 2D; Fig. S3D). Similar to many p53 agonists, ZDHHC1 extended the short half-life of wild-type p53 protein under protein synthesis inhibitor cycloheximide (CHX) treatment (Fig. 2E-F). These findings indicate that $\mathrm{ZDHHC1}$ plays an important role in the p53 signaling pathway through multiple means of regulation, including the possibility of p53 being a substrate for the acyltransferase based on the Co-IP results.

ZDHHC1 promotes p53 signaling through p53 palmitoylation In a previous study, Cao and colleagues proposed that protein palmitoylation is required for p53-mediated DNA damage response, but no specific enzyme was identified [15]. Our data demonstrated a substantial correlation between ZDHHC 1 and p53, including protein interaction. To verify if p53 is a target of ZDHHC1-mediated S-palmitoylation, we first utilized the palmitoylation site prediction tool CSS-PALM2.0 (http://www.csspalm. biocuckoo.org/). Among the potential cysteine residues within p53, C135, C176, C182, C275, and C277 have the highest scores (Fig. 3A). We then examined if the p53-ZDHHC1 interaction 

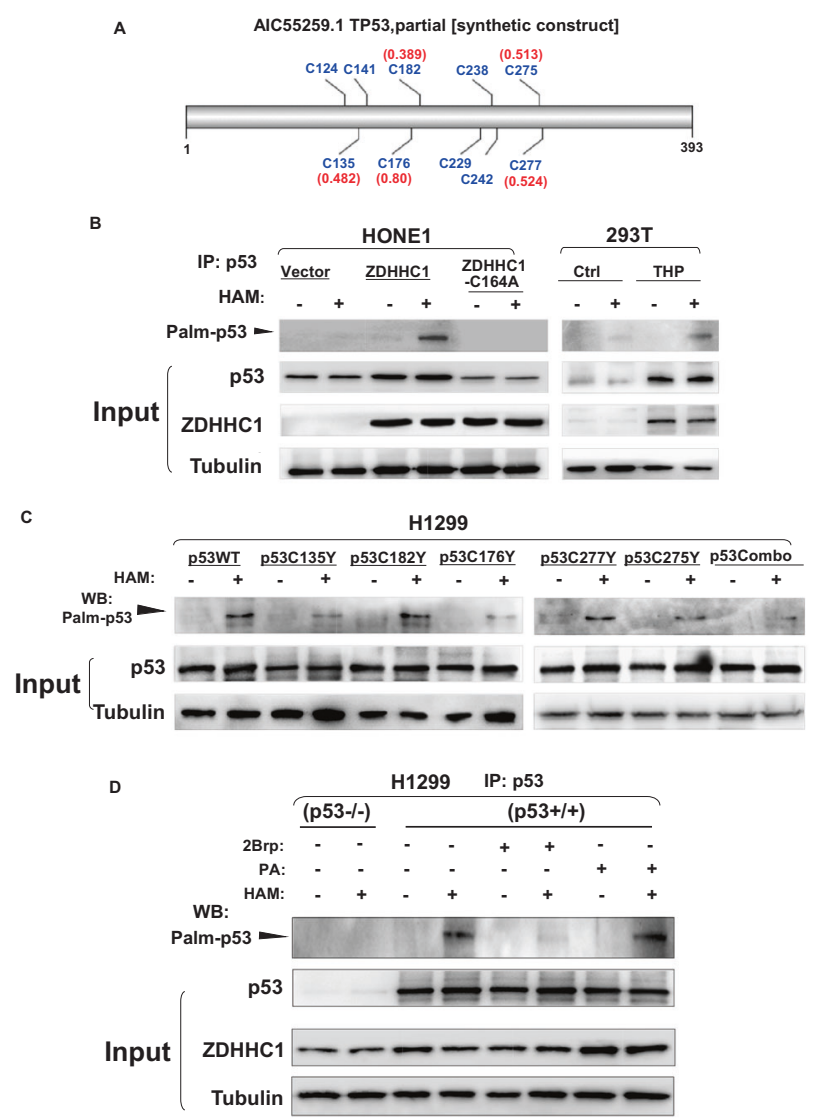

Fig. 3 ZDHHC1 mediates p53 palmitoylation. A Predicted cysteine residues on p53 susceptible to S-palmitoylation. B IP-ABE analysis of p53 palmitoylation in HONE1 and in 293T cells were treated with pirarubicin (THP). C IP-ABE analysis of p53 palmitoylation in $\mathrm{H} 1299$ cells when ectopically expressing p53 $3^{\mathrm{WT}}$ or p53 harboring specific mutations. D IP-ABE analysis of p53 palmitoylation in H1299 cells when treated with palmitoylation inhibitor $2 \mathrm{Brp}$ or promoter PA. HAM hydroxylamine, 2Brp 2-bromopalmitate, PA Palmitic acid.

resulted in p53 palmitoylation. Co-IP coupled with ABE assay was performed [16] and indeed, palmitoylated p53 was detected in HONE1 cells, but only when ZDHHC1 was ectopically expressed (Fig. 3B). As the DHHC motif is essential for the catalytic activity of palmitoyl-transferases, a mutation (C164A) was introduced to the DHHC motif in ZDHHC1, which abolished p53 palmitoylation (Fig. 3B). Both p53 and ZDHHC1 protein expression elevated when 293 T cells were treated with pirarubicin (THP), along with p53 palmitoylation (Fig. 3B), suggesting the form of lipidation is involved in p53's response to cellular stress. When the aforementioned p53 cysteine residues were respectively mutated and ectopically expressed in $\mathrm{H} 1299$ cells, the level of p53 palmitoylation decreased compared to the $\mathrm{p} 53^{\mathrm{WT}}$ group (Fig. $3 \mathrm{C}$ ). This effect accumulated when all five forms of mutated p53 are ectopically expressed together (p53Combo). The increase in palmitoylated p53 was almost completely negated and slightly enhanced in the presence of palmitoylation inhibitor 2-bromopalmitate (2Brp) and palmitic acid (PA) respectively (Fig. 3D). Taken together, the results indicated that p53 is susceptible to ZDHHC1-mediated palmitoylation at certain cysteine residues, which expanded upon the findings of Cao's group and the current understanding of p53 PTMs.

\section{ZDHHC1-mediated p53 palmitoylation is a prerequisite for p53 nuclear translocation}

Besides palmitoylation, one of the most consequential forms of protein modification for $\mathrm{p} 53$ is phosphorylation as it dictates the nuclear translocation of the transcription factor. We were interested to see if palmitoylation could influence the phosphorylation pattern or the subcellular localization of $\mathrm{p} 53$. We ectopically expressed the different forms of mutated p53 in $\mathrm{H} 1299$ cells. The phosphorylation of p53 at S9, S15, or S392 decreased in the single mutation groups and the p53Combo group compared with the p53 ${ }^{\mathrm{WT}}$ group (Fig. 4A, B), especially when C135, C176, and C275 were substituted. There was no observable change in other potential phosphorylation sites including S6, S20, S46, T18, and T81 (data not shown). Immunofluorescent staining showed that in $\mathrm{H} 1299-\mathrm{p} 53^{\mathrm{WT}}$ cells, p53 can accumulate in the nucleus (Fig. 4C). However, when the cysteine residues susceptible to palmitoylation were mutated into tyrosine, alanine, or serine residues, especially at the three sites that affected phosphorylation the most (C135, C176, and C275), nuclear translocation was significantly hindered (Fig. 4D; Fig. S4, right panel). Mutation of other cysteine residues did not stop p53 from entering the nucleus (Fig. S4, left panel) suggesting ZDHHC1mediated p53 palmitoylation plays an important role in p53 phosphorylation and subcellular localization. Blocking palmitoylation with 2 Brp also effectively suppressed the accumulation of $\mathrm{p} 53$ in the nucleus while PA had the opposite effect (Fig. 4E). Mutation of ZDHHC1 also retained p53 in the cytoplasm regardless of extrinsic palmitoylation regulators (Fig. 4E). Neither p53 ${ }^{\mathrm{WT}}$ nor p53Combo could abundantly accumulate in the nuclei of HONE1 cells when ZDHHC1 expression was not restored (Fig. 5A). These results further substantiate the necessity of ZDHHC1 in maintaining p53 nuclear translocation.

Next, we put cells under stress by treating cells with THP and obtained very similar results. The expression of ZDHHC1 and p53 in 293 T cells were triggered by THP treatment, and as suggested earlier, p53 mainly aggregated in the nucleus in the presence of ZDHHC1 (Fig. 5B). When the same treatment was applied to H1299 cells, ectopic p53 ${ }^{\mathrm{WT}}$ but not p53Combo was able to translocate to the nucleus (Fig. 5C).

It was reported that $\mathrm{p} 53$ could be oxidized by hydrogen peroxide $\left(\mathrm{H}_{2} \mathrm{O}_{2}\right)$ on zinc ion chelating amino acids $\mathrm{H} 179, \mathrm{C} 238, \mathrm{C} 242$, and $\mathrm{C} 176$ which alters the conformation and abolishes sequence-specific DNA binding of p53 [17]. However, treating cells with low or high concentrations of $\mathrm{H}_{2} \mathrm{O}_{2}$ did not affect the nuclear localization of p53 (Fig. 5C). p53 did not accumulate in the cell nucleus when ZDHHC1 expression was absent (MCF7, EC109, and HONE1) (Fig. 6, left panel) or when the $\mathrm{C} 176$ residue was mutated (H2122 and H1651) (Fig. 6, right panel). Mutations at other sites including other Cys residues (TE-10, CFPAC1, MB231, and KYSE150) did not stop p53 from entering the nuclei (Fig. 6, right panel). All of the above results indicate that ZDHHC1-mediated palmitoylation at specific cysteine residues was required for $\mathrm{p} 53$ nuclear translocation.

\section{ZDHHC1-mediated p53 palmitoylation is required for inhibiting tumor growth}

We have demonstrated that p53 nuclear translocation is heavily dependent on ZDHHC1-mediated palmitoylation. So next we tried to determine if ZDHHC1-mediated p53 palmitoylation translates into phenotypic changes. Consistent with our previous observations, ZDHHC1 efficiently inhibited MCF7 and HONE1 proliferation (Fig. 7A), partly through inducing cell cycle arrest (Fig. 7B; Fig. S5A) and apoptosis (Fig. 7C; Fig. S5B). ZDHHC1-C164A lost such effect along with its functional $\mathrm{DHHC}$ motif which is necessary for the palmitoylation of substrates such as p53 (Fig. 7A-C; Fig. S5A-B). Inhibiting palmitoylation overall with $2 \mathrm{Brp}$ also reversed the inhibitory effect of ZDHHC1 on cell proliferation (Fig. 7D, E).

As we described earlier, p53 mutations at the C135, C176, and C275 residues had the strongest inhibition effect on ZDHHC1mediated palmitoylation, phosphorylation, and nuclear translocation. Single mutated forms of ectopic p53 and p53Combo almost completely lost the ability to promote the expression of P21 and BAX, or suppress $\mathrm{H} 1299$ proliferation and induce apoptosis (Fig. 7F-l; Fig. S6A-C; Fig. S7). These results suggest that 


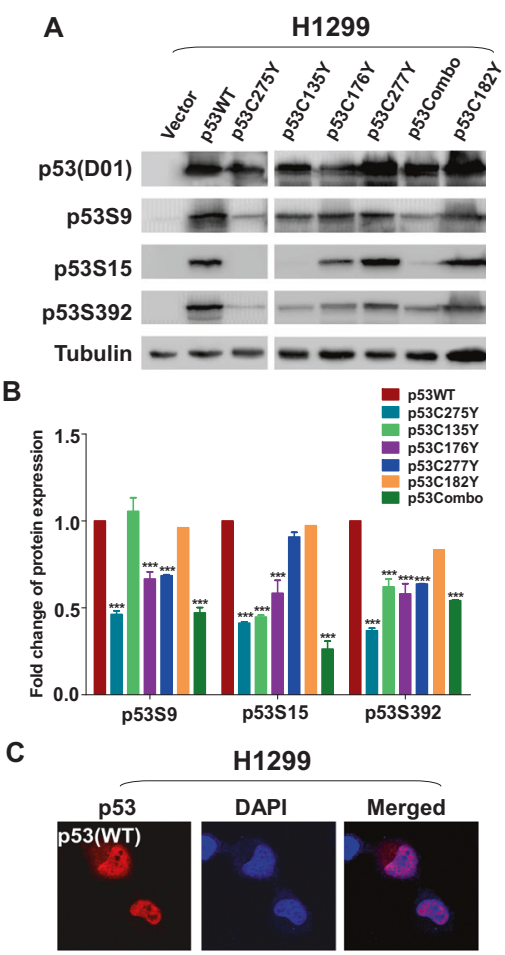

D

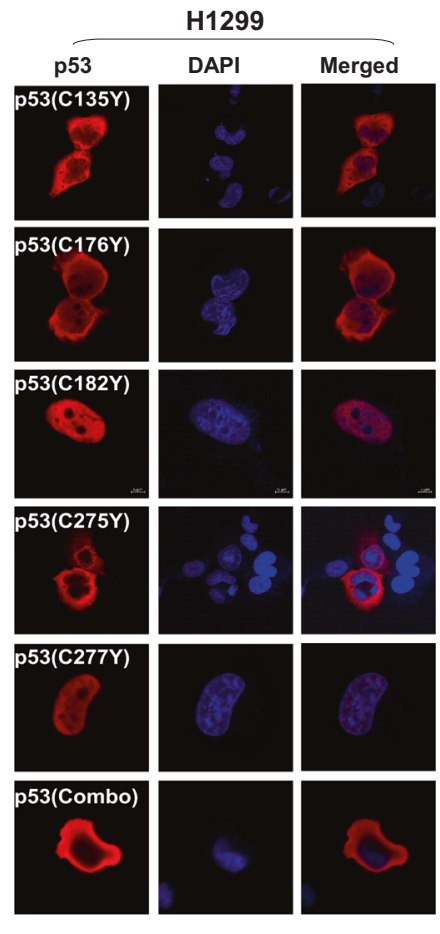

E

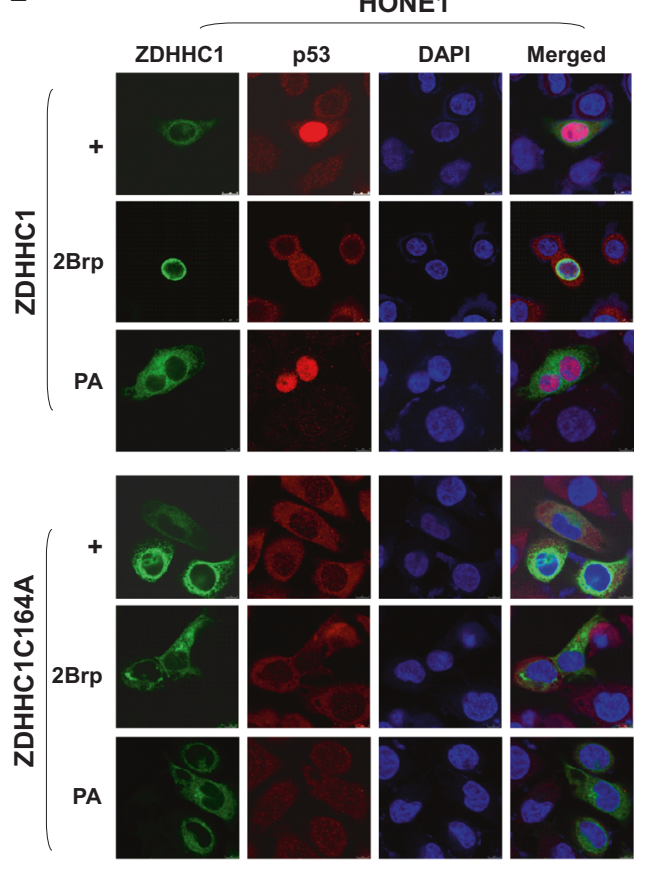

Fig. 4 ZDHHC1 mediates p53 phosphorylation and nuclear translocation. A Western blot analysis of the correlation between site-specific p53 mutations and p53 phosphorylation. B Quantification of the Western blot results in Fig. 4A. C Subcellular localization of ectopic p53 ${ }^{\text {WT }}$ in H1299 cells. D Subcellular localization of ectopic p53 with specific Cys substitutions in H1299 cells. E Subcellular localization of ZDHHC1 (top) or ZDHHC1-C164A (bottom) and p53 in HONE1 cells when treated with 2 Brp or PA. Values represent mean \pm SEM. ${ }^{*} p<0.05$. ${ }^{* * *} p<0.001$. $2 B r p$ 2-bromopalmitate, PA Palmitic acid.

C

A

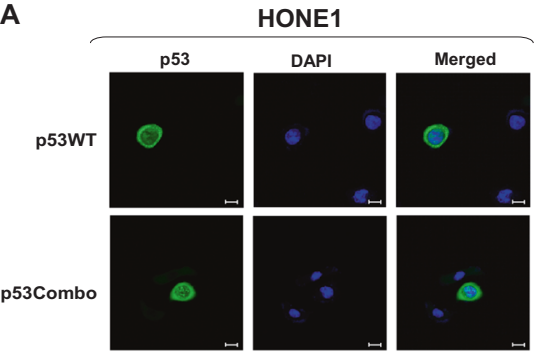

B

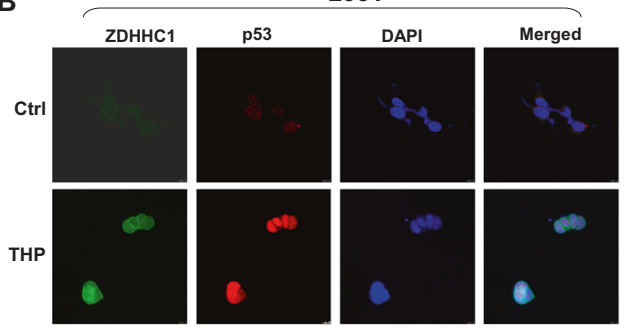

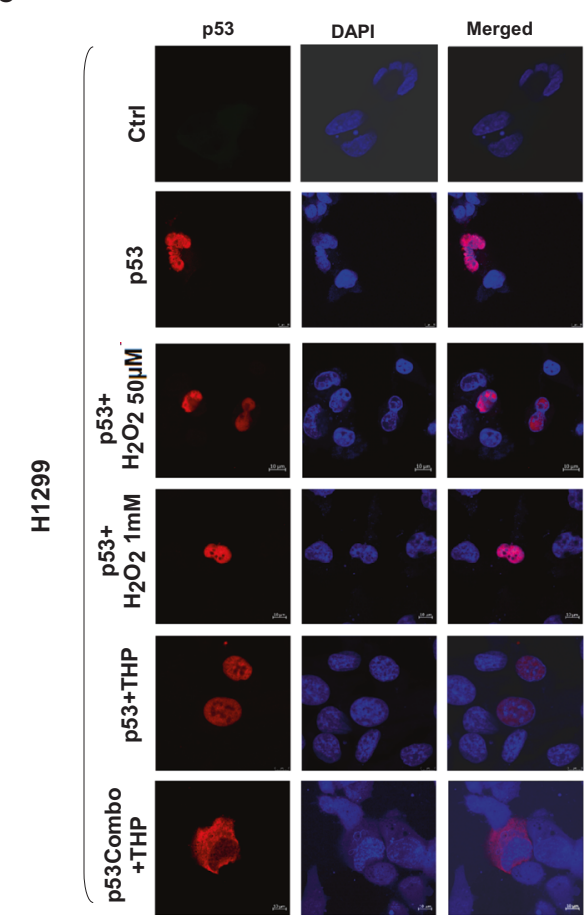

Fig. 5 ZDHHC1 is necessary for p53 protein translocation from cytosol to nucleus. A Subcellular localization of p53 ${ }^{\mathrm{WT}}$ and $\mathrm{p} 53 \mathrm{Combo}$ in HONE1 cells by immunofluorescent staining. B Subcellular localization of ZDHHC1 and p53 in 293 T cells after THP treatment by immunofluorescent staining. C Subcellular localization of ectopic p53 or p53Combo in $\mathrm{H} 1299$ cells after THP or $\mathrm{H}_{2} \mathrm{O}_{2}$ treatment by immunofluorescent staining. Pictures were photographed using confocal microscopy. THP: Pirarubicin. 


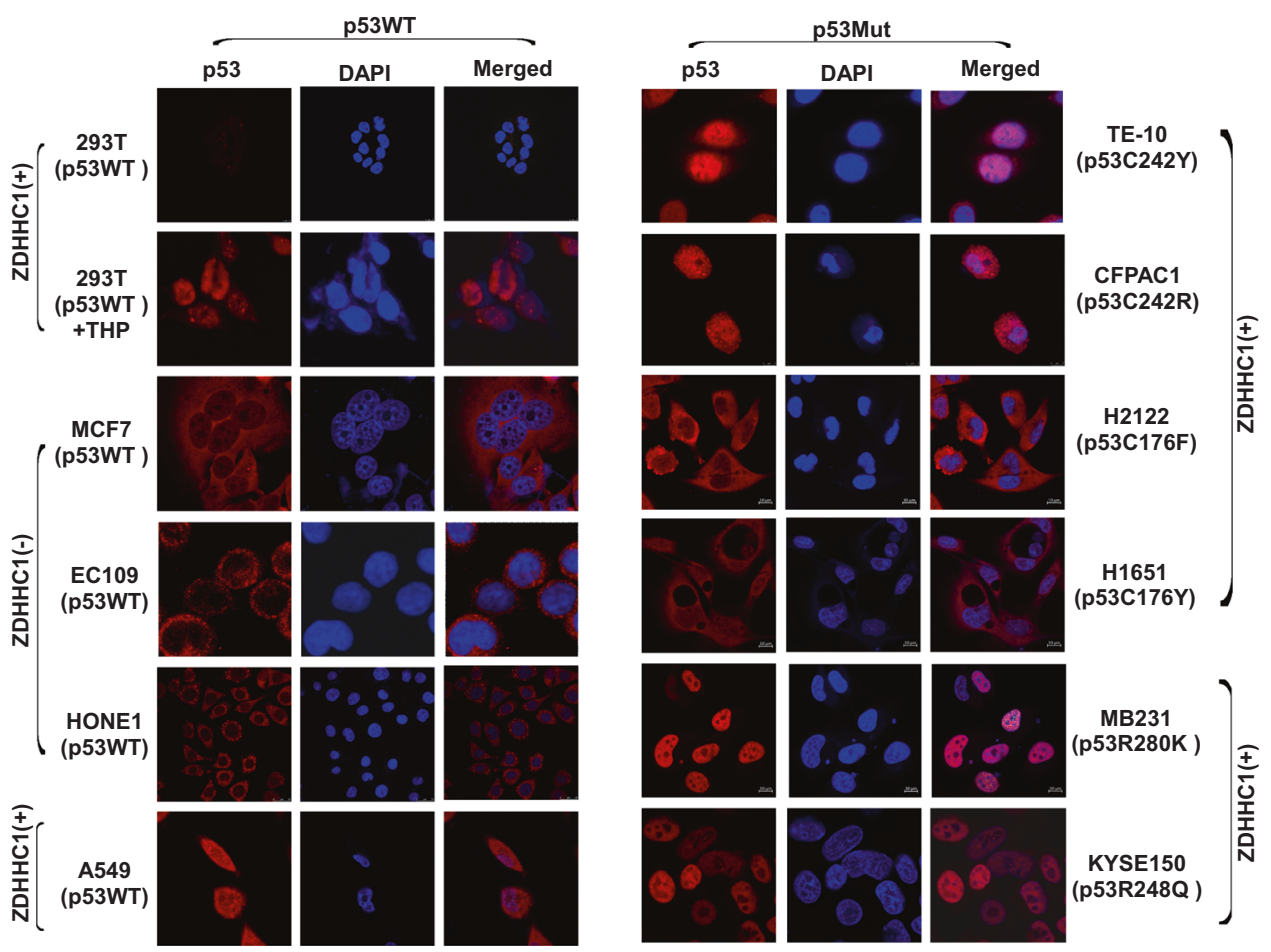

Fig. 6 ZDHHC1 and p53 mutations determine p53 localization. (Left) Indirect immunofluorescent staining of p53 ${ }^{\mathrm{WT}}$ in $293 \mathrm{~T}$ (with or without THP treatment), MCF7, EC109, HONE1, and A549 cells. (Right) Indirect immunofluorescent staining of p53 in cells carrying varied mutant p53. TE10 (p53-C242Y), CFPAC1 (p53-C242R), H2122 (p53-C176F), H1651 (p53-C176Y), MB231 (p53-R280K), and KYSE150 (p53-R248Q) were enlisted as experimental subjects. Pictures were photographed using confocal microscopy. THP: Pirarubicin.

ZDHHC1-mediated p53 palmitoylation is a key mechanism for ZDHHC1's tumor-suppressing functions.

\section{ZDHHC1 promoter hypermethylation is mediated by $\mathrm{p} 53^{\mathrm{WT}}$. recruited DNMT3A}

We previously found that the $Z D H H C 1$ promoter is hypermethylated in many cancers, causing the frequent lack of expression [13]. The silencing of $Z D H H C 1$ expression in the presence of promoter methylation and $\mathrm{p} 53^{\mathrm{WT}}$ respectively made us wonder if these events were correlated. Methylation-specific PCR (MSP) was conducted to examine the methylation status of ZDHHC1 promoter in an array of human tumor cells. While most of the cell lines with wild-type TP53 tested positive for methylation, none of the cell lines with mutated TP53 did (Fig. 8A). When we separated human breast cancer samples by the state of p53 status, all 5 of the p53 $3^{\text {mut }}$ samples tested negative for $Z D H H C 1$ promoter methylation. Most p53 $3^{\mathrm{WT}}$ samples were positive for methylation, only 2 out of 29 samples were unmethylated (Fig. 8B). We found frequent mutations in exon 7 of TP53 in the unmethylated samples (Fig. S8). Exons 5, 6, and 8 also frequently harbor TP53 mutations, but in these samples, no mutations were found in these regions (Fig. S8).

In H1299 cells, the mRNA expression of ZDHHC1 is significantly suppressed by ectopic $\mathrm{p} 53^{\mathrm{WT}}$ due to decreased transcriptional activity, but p53Combo failed to achieve similar effects (Fig. 8C, D). The same results were also observed in MCF7 cells (Fig. 8E). ZDHHC1 promoter activity was restored with DNA methylation inhibitor 5-aza2'-deoxycytidine (Aza) (Fig. 8F), confirming our previous findings [13]. To identify the specific epigenetic regulators, we used ChIP-qPCR to analyze the promoter region of $Z D H H C 1$. Results showed that p53 colocalized with DNMT3A at the potential p53 binding site, but not other DNA methyltransferases (Fig. 8G). DNMT3A inhibited ZDHHC1 promoter activity in a dose-dependent manner (Fig. 8H). ChIP-qPCR was repeated after Aza demethylation treatment and the localized binding of DNMT3A significantly decreased (Fig. 8I). DNA damage by
THP promoted the enrichment of both p53 and DNMT3A (Fig. 8J). We took a closer look at the promoter region of ZDHHC1. We found a potential p53 binding site next to a CpG island (Distal) and p53 binding sites located in close proximity to the ATG codon (Proximal). We designed ChIP-qPCR primers for both regions and p53/DNMT3A enrichment was only found at the distal site (Fig. 8K). On the p53 binding site (distal) near the CpG island, HDAC1 was also enriched under DNA damage (Fig. 8K). These results indicate that epigenetic regulation of $Z D H H C 1$ expression by $\mathrm{p} 53$ is not limited to DNA methylation, but also involve other mechanisms such as histone deacetylation. Taken together, these data point to a negative epigenetic feedback loop between p53 and ZDHHC1 (Fig. 9) which provides clarification for previously reported findings and a possible mechanism for evasion of p53 tumor surveillance.

\section{DISCUSSION}

As the most prominent tumor suppressor gene, TP53 had been under the spotlight for almost 4 decades [18]. However, the clinical application based on this celebrity gene is fairly limited due to the complexity of its regulatory network on multiple levels. Here we report a novel form of post-translational modification for p53 that is catalyzed by the palmitoyl S-acyltransferase (PAT) ZDHHC1 (zinc finger DHHC-type containing 1).

Post-translational modifications are common and crucial for p53, among which ubiquitination, phosphorylation, and acetylation are the most extensively studied [19]. Mdm2-p53 interaction has been well-established as the central node of the p53 pathway, and such interaction builds upon p53 ubiquitination [18]. When cells were introduced to certain forms of stress such as DNA damage, the Mdm2-p53 interaction moves in a direction in which p53 is phosphorylated and freed from Mdm2's control. While phosphorylation-mediated stabilization and nuclear translocation as well as ubiquitination-mediated proteasomal degradation are 
A

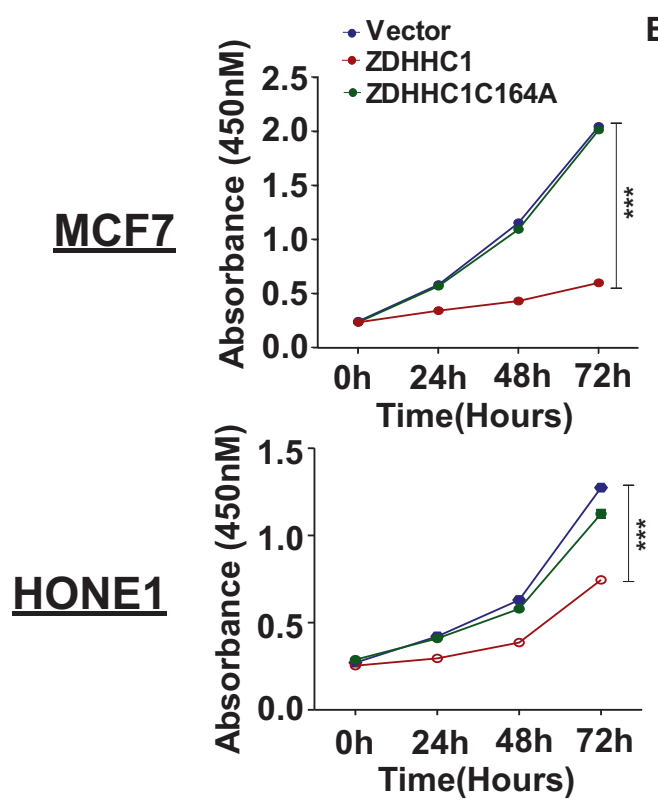

B
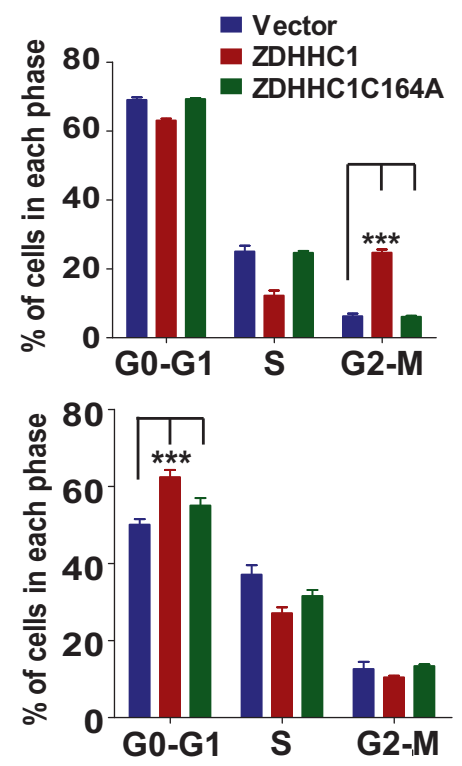

C
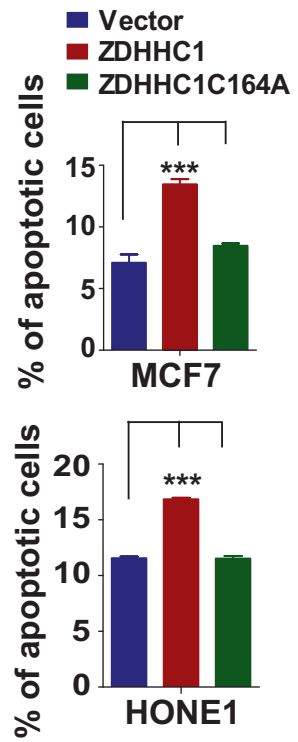

D

G

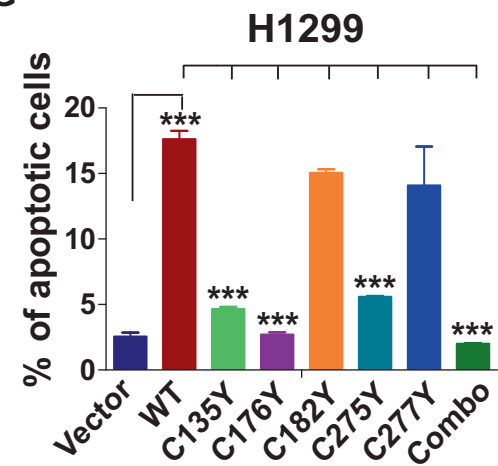

E

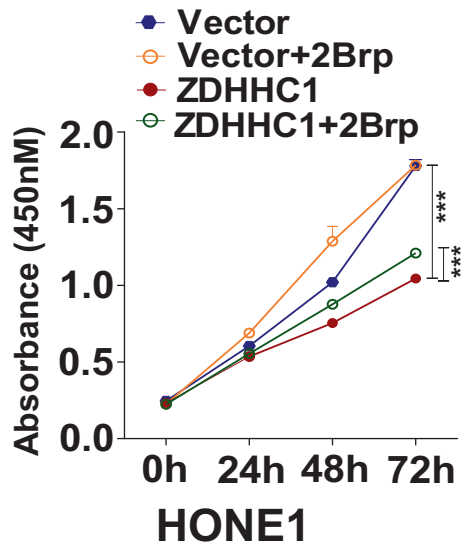

H

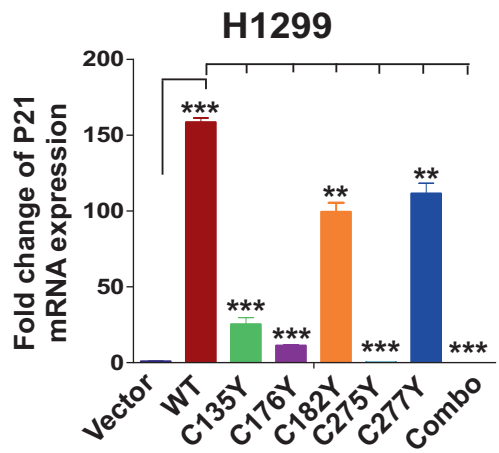

F
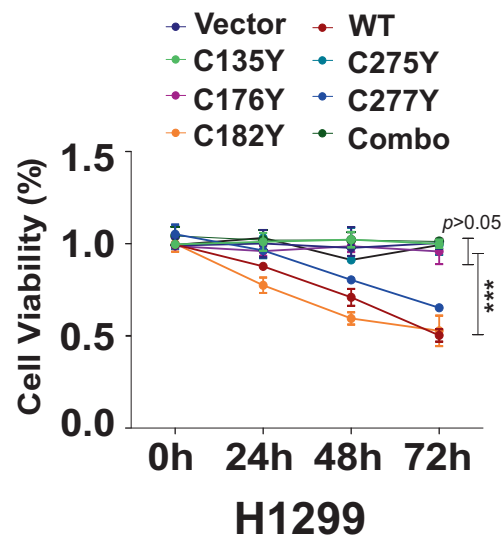

I

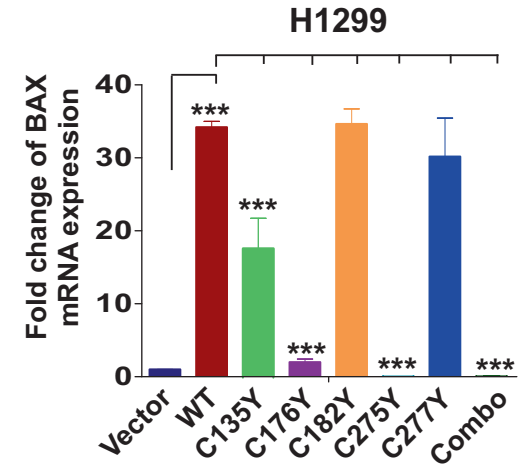

Fig. 7 ZDHHC1-mediated p53 palmitoylation is required for suppressing tumor cell growth. A CCK8 proliferation assay of MCF7 and HONE1 cells. B Cell cycle analysis of MCF7 and HONE1 cells. See also Fig. S5A. C Flow cytometric analysis of apoptotic MCF7 and HONE1 cells expressing ZDHHC1 or ZDHHC1C164A. See also Fig. S5B. D CCK8 proliferation assay of MCF7 cells treated with 2Brp. E CCK8 proliferation assay of HONE1 cells treated with 2Brp. F CCK8 proliferation assay of $\mathrm{H} 1299$ cells ectopically expressing different variants of p53. G Flow cytometric analysis of apoptotic $\mathrm{H} 1299$ cells ectopically expressing different variants of p53. See also Fig. S6A-C. H mRNA expression of $P 21$ in H1299 cells by qRT-PCR. I mRNA expression of $B A X$ in H1299 cells by qRT-PCR. Values represent mean \pm SEM. ${ }^{* *} p<0.01,{ }^{* *} p<0.001$.

major events for regulating p53 activity, there have been conflicting reports on the roles of other types of PTM [20].

Palmitoylation, or S-acylation, is a major type of reversible protein lipidation that is highly conserved in eukaryotic organisms [21].
The process of palmitoylation is mainly mediated by the $\mathrm{zDHHC}$ family, which links two of the most important macromolecules through covalent thioester bonds, altering the physical and chemical characteristics of proteins. Thousands of proteins could be susceptible 


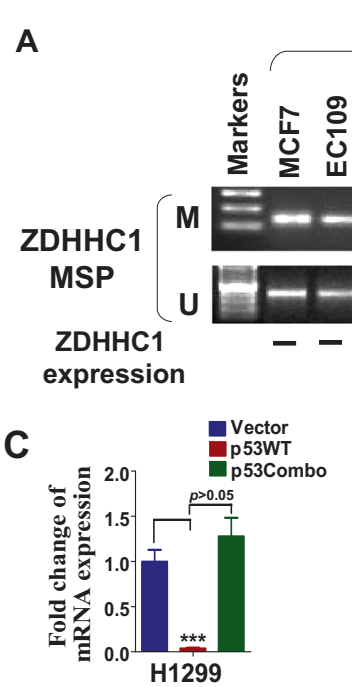

G

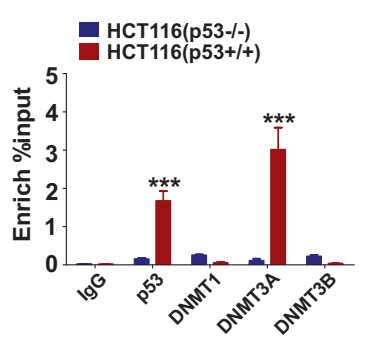

p53WT

p53Mut
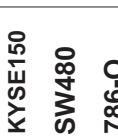

\section{:}

B

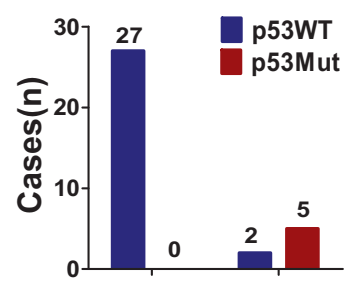

ZDHHC1 Methylation Unmethylation

D

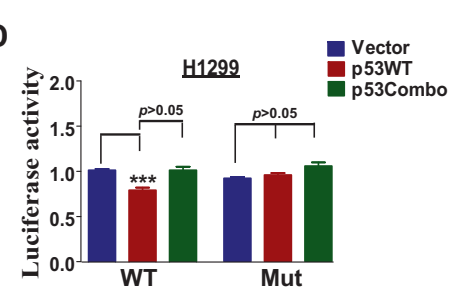

E

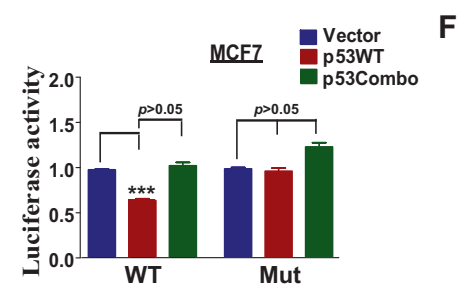

$F$

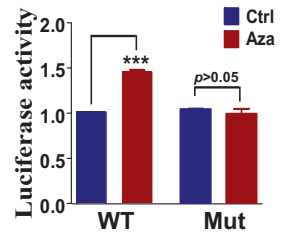

H

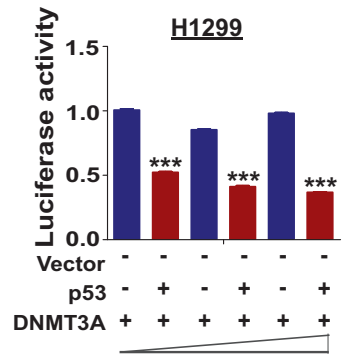

I

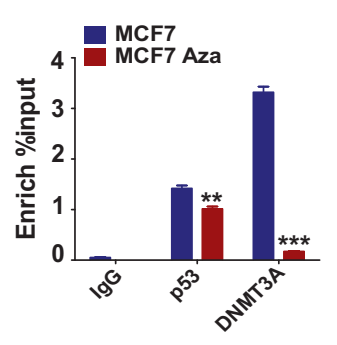

J

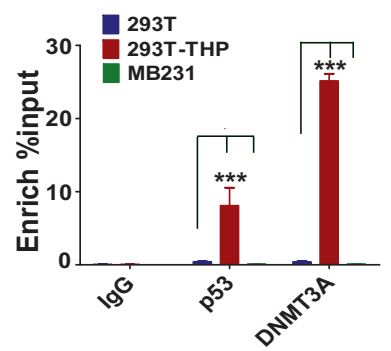

K
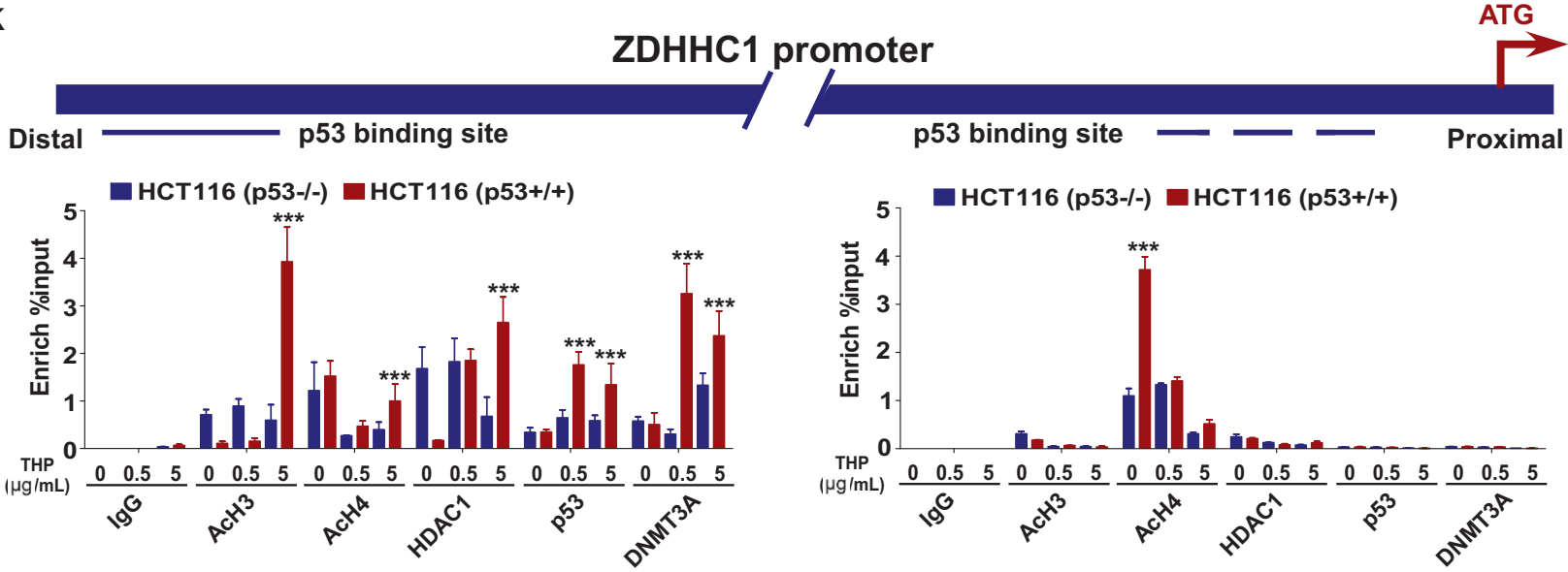

Fig. 8 ZDHHC1 methylation correlates with p53 mutation, and p53 recruits DNMT3A to ZDHHC1 promoter to inhibit transcription. A ZDHHC1 promoter methylation in human cancer cell lines grouped by p53 mutation status. B Correlation between ZDHHC1 promoter methylation and p53 mutation in 34 human breast cancer tissue samples. C mRNA expression of ZDHHC1 in H1299 cells ectopically expressing p53 $3^{\text {WT }}$ or p53Combo. D ZDHHC1 promoter activity with or without mutating the p53 binding site in $\mathrm{H} 1299$ cells. E ZDHHC1 promoter activity with or without mutating the p53 binding site in MCF7 cells. F ZDHHC1 promoter activity with or without mutating the p53 binding site in MCF7 cells treated with Aza. G Enrichment of p53, DNMT1, DNMT3A, and DNMT3B near the p53 binding site on the ZDHHC1 promoter by ChIP-qPCR. H ZDHHC1 promoter activity in H1299 cells with ectopic p53 expression and different levels of ectopic DNMT3A expression. I Enrichment of p53 and DNMT3A near the p53 binding site on the ZDHHC1 promoter before and after MCF7 cells were treated with Aza. J Enrichment of p53 and DNMT3A near the p53 binding site on the ZDHHC1 promoter in 293 T cells treated with THP. MB231 cells were used as a control. K Enrichment of epigenetic regulators near the distal and proximal p53 binding sites on the ZDHHC1 promoter. Values represent mean \pm SEM. ${ }^{* *} p<0.01,{ }^{* *} p<0.001$.

to palmitoylation, implying the deep impact of such modification in biological and pathological settings [22]. Palmitoylation of p53 hasn't been reported but two studies hinted at the possibility. In the first study, inhibiting palmitoylation with 2 Brp effectively blocked mouse osteoblast differentiation, but not in $p 53^{-\%}$ cells [23]. DNA damage-induced p53 activation in mouse embryonic fibroblasts could also be hindered by 2Brp [15]. The ZDHHC1-p53 protein interaction and the covalent attachment of palmitoyl groups on p53 cysteine residues may provide one plausible explanation, among many, for the effect of $2 \mathrm{Brp}$ on p53 activation.

The addition of lipids alters the protein's affinity for water, therefore affecting the protein's intracellular localization [24]. Our results indicate that palmitoylated Cys residues reduced the amount of substrate proteins in the cytoplasm and concentrated in the 


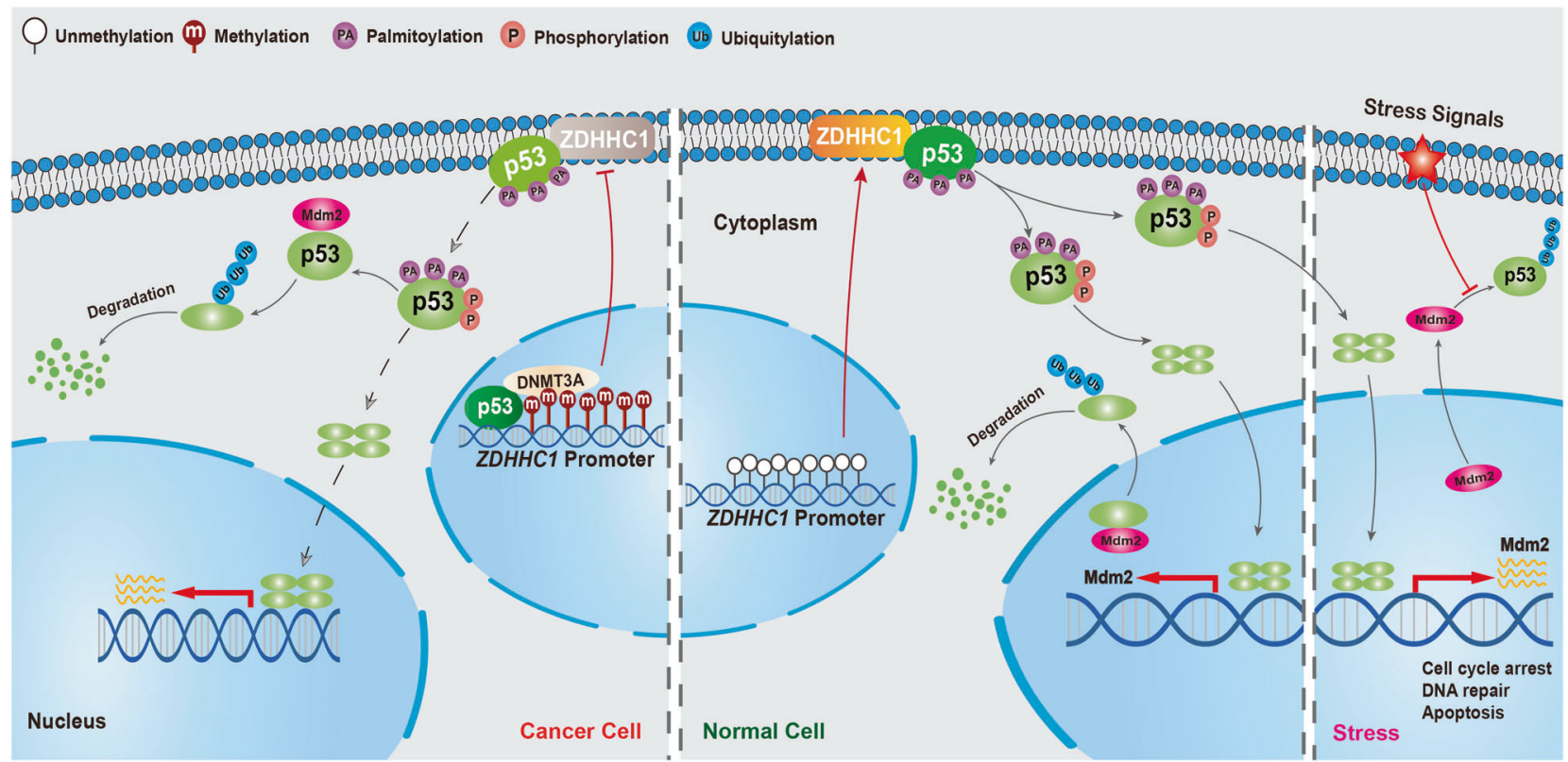

Fig. 9 Graphic description of the epigenetic regulatory loop formed by ZDHHC1, p53, and DNMT3A. ZDHHC1 interacts with p53 and mediates the attachment of palmitic acids to certain cysteine residues of the tumor suppressor, which is required for the subsequent phosphorylation and nuclear translocation that keeps cells in check when facing stress signals. This mechanism is shut off in many cancer cells due to the binding of the p53-DNMT3A complex to the CpG-rich region of ZDHHC1 promoter and epigenetically silencing ZDHHC1 expression, thus forming a negative regulatory loop that restricts p53 from entering the nucleus and releases the cell from p53's control.

nucleus, yet there is no evidence showing palmitoylation affects p53's affinity to nuclear membranes. It has been reported that importin alpha can be palmitoylated and bind to the plasma membrane, decreasing its level in the cytoplasm [25]. The opposite direction of transport between p53 and importin alpha could possibly be explained by the difference in the palmitoylationphosphorylation interaction. Lipidation of p53 led to increased levels of phosphorylation at multiple sites while phosphorylation hindered importin a palmitoylation. Interestingly, importin alpha itself is a crucial regulator of protein nuclear transport [26]. Therefore, we speculate ZDHHC1-mediated palmitoylation enhanced p53's affinity for the lipid bilayer that forms the nuclear envelope, thus creating a more friendly microenvironment for the nuclear import of p53, possibly with the involvement of importin alpha itself.

As our data suggested, C135, C176, and C275 out of the 5 candidate Cys residues are the most likely targets of ZDHHC1mediated palmitoylation, yet these residues are also involved in the regulation of p53 activity through alternative mechanisms. C135 and C176 also enable the binding of p53 to NM23-H1 and STRAP respectively, resulting in the disassociation of $\mathrm{p} 53-\mathrm{Mdm} 2$ complex and p53 stabilization [27]. Oxidation of $\mathrm{C} 176$ by $\mathrm{H}_{2} \mathrm{O}_{2}$ was shown to inhibit the DNA binding ability [28]. But in our case, $\mathrm{H}_{2} \mathrm{O}_{2}$ didn't seem to interfere with palmitoylation and nuclear transport. C275 mutation was found in lung cancer tissues [29] while it was the only 1 of 8 cysteine residues within p53 that affected the promoter binding affinity of Gadd45. Our results revealed that ZDHHC1 not only modulates the palmitoylation of $\mathrm{p} 53$ but also impacts the phosphorylation at multiple residues. The effect on p53 phosphorylation is likely one of the mechanisms by which ZDHHC1 promotes the transportation of $\mathrm{p} 53$ into the nucleus.

We have previously identified promoter DNA hypermethylation as a major cause of the silencing of ZDHHC1 in tumors [13]. Now we have reason to believe that p53 plays a critical role in causing this epigenetic event, which completes the negative feedback loop. Our data showed that p53 can bind to the promoter of $Z D H H C 1$, mostly next to a CpG island, and recruits the epigenetic writers DNMT3A and HDAC1 to the region to silence ZDHHC1 expression. In a few $\mathrm{p} 53^{\mathrm{WT}}$ cell lines (A549, HCT116, and HepG2), the promoter region of $Z D H H C 1$ was unmethylated, which could be explained by their unique genetic and/or epigenetic backgrounds that could affect the $\mathrm{ZDHHC1}$ promoter methylation process. A similar trend can be seen in breast tumor tissue samples, each of its own heterogenous characteristics. 2 out of 29 p53 WT tissue samples tested in this study fall out of the box by being negative for ZDHHC1 promoter methylation. We propose DNMT3A to be the major epigenetic writer in this process, but other factors could also be at play. For example, HepG2 harbors a DNMT3B mutation [30] which may interfere with the de novo DNA methylation process. To a certain extent, this negative feedback loop could protect the tumor cells from the synergistic anti-tumor effect of p53 and ZDHHC1, and be one of the reasons why many tumors don't bear TP53 mutations yet still thrive.

From the clinical point of view, the dependent lethality of p53 and ZDHHC1 translates into differences in prognosis. Many chemotherapy reagents eradicate tumor cells by inducing DNA damage and subsequent pathway activation. Through the negative feedback of $\mathrm{p} 53$ and $Z D H H C 1$, there may be potential for predicting chemosensitivity by detecting the level of $Z D H H C 1$ promoter methylation, which can be easily achieved using both solid and liquid biopsy samples. The biomarker value of $Z D H H C 1$ will need further validation with more data on clinical samples, which we are collecting and will be thoroughly examined. Therapeutics that target palmitoylation is also still lacking due to insufficient knowledge on the detailed mechanisms. Contradicting evidence suggests that palmitoylation may need to be precisely targeted in different types of malignancies and in distinct genetic backgrounds [31].

Based on our results, we propose palmitoylation as a novel form of post-translational modification for p53, which is mediated by ZDHHC1 (Fig. 9). Palmitoylation is necessary for p53 nuclear trafficking and subsequent pathway activation. In turn, p53 regulates $\mathrm{ZDHHC1}$ expression epigenetically by recruiting DNMT3A and HDAC1 to the promoter region. The regulatory feedback loop could help explain the development of p53wildtype cancers and may be utilized for the advance of p53 targeted therapy. 


\section{MATERIALS AND METHODS}

Cell lines, tumor samples, and normal tissues

Cell lines (HONE1, MCF7, A549, EC109, HCT116, HCT116 ${ }^{\text {TP53-- }}$, TE-10, CFPAC1, H2122, H1651, MB231, KYSE150, and H1299) were provided by ATCC (American Type Culture Collection, Manassas, VA, USA) or its collaborators. All cell lines were cultured in RPMI1640 (Gibco-BRL, Karlsruhe, Germany) media in general use and regularly tested for mycoplasma contamination. All cell lines were either recently purchased or recently authenticated by STR profiling. All tissues samples were collected from the First Affiliated Hospital of Chongqing Medical University, including all the primary tumor tissues and adjacent normal tissues. Certified pathologists verified that at least $70 \%$ of the cells in tumor samples are tumor cells. This research was authorized by the Institutional Ethics Committees of the First Affiliated Hospital of Chongqing Medical University (Approval notice: \# 2016-61) and complied with the Declaration of Helsinki. Informed consent was obtained from all patients

\section{Reverse transcription-PCR and Quantitative real-time PCR}

RNA was isolated from samples using Trizol according to the manufacturer's introductions. DNA was extracted with QIAamp DNA mini kit (Qiagen, Hilden, Germany). In RT-PCR, the total volume of each reaction mixture was $10 \mu \mathrm{l}$ with $2 \mu \mathrm{l}$ of $\mathrm{CDNA}$, and GAPDH was used as the inner control. The sequence of primers is presented in table S1. RT-PCR conducted using the Go-Taq (Promega, Madison, WI, USA) system under the condition of 32 cycles for samples, and 23 cycles for GAPDH. Quantitative real-time PCR (qRT-PCR) was conducted on the HT7500 System (Applied Biosystems, US) following the unit manual. The denaturing, annealing, and extension temperatures were set at 95, 60, and $72{ }^{\circ} \mathrm{C}$, respectively.

\section{Bisulfite conversion and methylation-specific PCR (MSP)}

Genomic DNA was obtained from cells and tissues. Bisulfite conversion of DNA samples was conducted as previously reported [32]. The primers used for MSP were listed in table S1. AmpliTaq-Gold DNA Polymerase (Applied Biosystems, US) was employed to amplify target DNA. After electrophoresis on $2 \%$ agarose gels, the results were photographed by a gel imaging system (Bio-RAD Gel Doc XR +, USA). MSP analysis was performed as previously described [33].

\section{Construction of plasmids and stable cell lines}

Coding regions of target genes were inserted into the pEGFP-C1 framework plasmids. Recombinant plasmid had been verified by sequencing. After transfection, stable cell lines were selected using G418 (300 $\mathrm{\mu g} /$ $\mathrm{ml}$ for HONE1, $700 \mu \mathrm{g} / \mathrm{ml}$ for MCF7). For verification of ectopic gene expression, TRI reagent/protein extraction kit (Thermo Scientific, \#23225) was used to perform total RNA/protein extraction. DNA contamination was cleaned using DNase (Deoxyribonuclease, Ambion, Austin, TX, USA). Moreover, we performed RT-PCR to amplify target genes and Western Blot was used to confirm the protein expression. All plasmids utilized in the study are listed in table S2.

\section{Indirect immunofluorescent staining}

First, cells were seeded on glass coverslips and fixed with $4 \%$ paraformaldehyde in PBS. 0.1\% Triton X-100 in PBS was then used for permeabilization. $1 \%$ bovine serum album in PBS was used for blocking non-specific conjugation at room temperature. Subsequently, the primary antibodies were applied for incubation at $4{ }^{\circ} \mathrm{C}$ overnight, followed by the incubation of the DyLight-conjugated anti-rabbit or anti-mouse secondary antibodies at room temperature for $1 \mathrm{~h}$. Next, DAPI (4,6-diamidino-2phenylindole) was used to counterstain the nucleus at room temperature for $5 \mathrm{~min}$. Imagines were obtained under a fluorescence microscope.

\section{Tumor xenografts model in nude mice}

$5 \times 10^{6}$ cells in $0.1 \mathrm{ml}$ PBS were injected into the flanks of each female BALB/c nude mouse ( $n=6,4-6$ weeks old). During the following 16 days, a vernier caliper was utilized to measure the diameter of the tumor every three days. Tumor volume was calculated using the following formula: volume $=$ length $\times$ width $\wedge 2 \times 0.52$. The xenografts were removed after the mice had been sacrificed and then weighed. The number of mice used in this study was chosen based on the experience from previous experiments under similar conditions. The standard deviation of xenograft size (main outcome) was considered. The number of mice was chosen to ensure adequate power and to detect biologically relevant differences. No randomization was used in the experiments in the animal studies. Our experiments were approved by the Animal Experiment Ethics Committee of Chongqing Medical University.

\section{Flow cytometry}

Flow cytometric analysis of cellular apoptosis was conducted as previously described [13]. Briefly, cells were double-stained with Annexin Vfluorescein isothiocyanate (FITC; BD Biosciences, San Jose, CA) and PI according to the manufacturer's protocol. Cells were then analyzed using FACSCalibur $^{\mathrm{TM}}$ (BD Biosciences, San Jose, CA). Data were analyzed using the CellQuest ${ }^{\mathrm{TM}}$ software (BD Biosciences, San Jose, CA).

\section{Western blotting}

Western blot was conducted as previously described [34]. Briefly, cells were lysed in the lysis buffer containing a protease inhibitor cocktail. The lysate was centrifuged at $4{ }^{\circ} \mathrm{C}$ and $1000 \mathrm{~g}$ for $10 \mathrm{~min}$. The supernatant was harvested and quantified for sodium dodecyl sulfate-polyacrylamide gel electrophoresis (SDS-PAGE). The following primary antibodies were used in this study: anti-ZDHHC1 (\#ab223042, Abcam), anti-p53 (DO-1) (\#sc-126, Santa Cruz Biotechnology, Inc), or Phospho-p53 Antibody Sampler Kit (\#9919, Cell Signaling Technology), while Tubulin (\#sc-8035, Santa Cruz Biotechnology, Inc) and ACTB (\#sc8432, Santa Cruz Biotechnology, Inc) were used as the internal control. The secondary antibody incubation was set for $1 \mathrm{~h}$ at $37^{\circ} \mathrm{C}$. Enhance Chemiluminescence detection kit (Amersham Pharmacia Biotech, Piscataway, NJ, USA) was used for the detection and analysis of protein expression.

\section{Luciferase reporter assay}

During the construction of plasmids, the reporter was ligated in pGL3/Basic plasmid together with the target gene. PCR-amplified promoter regions and the reporter sequence were inserted into the pGL3/Basic plasmid using a seamless cloning kit (D7010S, Beyotime, Beijing, China). The plasmids were co-transfected with Renilla luciferase reporter pRL-TK (Promega) functioning as an internal control. Luciferase activity was measured after $48 \mathrm{~h}$ of transfection using a dual-luciferase reporter assay kit (Promega) following the manufacturer's instructions. All experiments were performed in triplicate.

\section{Co-immunoprecipitation (Co-IP) assay}

Cells were suspended in RIPA buffer and subsequently lysed via sonication. Protein quantitation was conducted using the BCA Protein Assay Kit (Pierce, Thermo Fisher Scientific, US) following the manufacturer's manual. To pre-clear the cell lysate, A-sepharose magnetic beads were used. After incubation at $4{ }^{\circ} \mathrm{C}$ for $30 \mathrm{~min}$, immunoprecipitating antibodies were then added into pre-cleared cell lysate. The immune complex was separated under the assistance of A-sepharose magnetic bead slurry. The beads were washed and the supernatant was used for immunoprecipitation (See Western blot methods).

\section{Co-IP-ABE assay}

Co-IP coupled with ABE (Co-IP-Acyl-Biotin-Exchange) assay was performed as previously described [35]. Cultured cells are lysed, a target protein is then purified using a target-specific antibody, and immobilized on sepharose beads coated with protein $\mathrm{G}$ or $\mathrm{A}$. The purified target protein is then treated with $\mathrm{N}$-Ethylmaleimide (NEM) to irreversibly bind and block free thiol (-SH) groups along unmodified cysteines (C). The target protein is then subjected to treatment with hydroxylamine (HAM), resulting in specific cleavage of thioester bonds at palmitoylated cysteines and the unmasking of a free palmitoylated thiol group (-SH). Next, the target protein is treated with a thiol-reactive biotin molecule, biotin-BMCC, resulting in specific biotinylation of the palmitoylated cysteine. Finally, the biotinylated target protein is eluted and removed from the antibody and beads. The target protein with its palmitoylated cysteine(s) tagged with biotin is now suitable for SDS-PAGE, and western blotting with streptavidin to detect for palmitoylation of the purified protein.

\section{Chromatin immunoprecipitation (ChIP)-qPCR assay}

ChIP was performed according to the manual of the SimpleChIP® Enzymatic Chromatin IP Kit (\#9003, Cell Signaling Technology) [34]. Crosslinking of protein-DNA complexes was achieved with $1 \%$ formaldehyde before lysis (See Western blot methods). The lysed samples were sonicated to shear the chromatin and then incubated overnight with 
antibodies (Anti-p53: Cat. \# 17-613, Millipore; Anti-acetyl-Histone H4: Cat. \# 06-866, Millipore; Anti-acetyl-Histone H3: Cat. \# 06-599, Millipore; AntiDnmt3a: ab2850, Abcam; Anti-Dnmt1: ab13537, Abcam; Anti-Dnmt3b: ab122932, Abcam) or normal lgG (\#2729, Cell Signaling Technology) at $4{ }^{\circ} \mathrm{C}$, followed by capture with protein A/G magnetic beads (\#9006, Cell Signaling Technology) for $2 \mathrm{~h}$. Then DNA was purified for the subsequent qPCR assays. ChIP-qPCR primers are listed in table S1.

\section{Bioinformatics and statistical analyses}

NCBI Blast program http://www.ncbi.nlm.nih.gov/BLAST/ was used to analyze specific CDNA sequences. p53 transcription factor binding sites on the ZDHHC1 promoter were determined by the Jaspar database (Jaspar. genereg.net). All the data acquired were analyzed using SPSS VERSION.6 (Chicago, IL, USA). Two-tailed Student's $t$-test, $X^{2}$ test, together with Fisher's exact test was used according to the specific circumstance. The variance was similar between the groups that were statistically compared using a $t$ test. Statistical significance was recognized when $p<0.05$.

\section{REFERENCES}

1. Hanahan D, Weinberg RA. Hallmarks of cancer: the next generation. Cell. 2011;144:646-74.

2. Gnanapradeepan K, Basu S, Barnoud T, Budina-Kolomets A, Kung CP, Murphy ME. The p53 Tumor Suppressor in the Control of Metabolism and Ferroptosis. Front Endocrinol. 2018;9:124

3. Olivier M, Hollstein M, Hainaut P. TP53 mutations in human cancers: origins, consequences, and clinical use. Cold Spring Harb Perspect Biol. 2010;2:a001008.

4. Brooks $\mathrm{CL}, \mathrm{Gu}$ W. Ubiquitination, phosphorylation and acetylation: the molecular basis for p53 regulation. Curr Opin Cell Biol. 2003;15:164-71.

5. Ito A, Lai C, Zhao X, Saito S, Hamilton M, Appella E, et al. p300/CBP-mediated p53 acetylation is commonly induced by $\mathrm{p} 53$-activating agents and inhibited by MDM2. EMBO J. 2001;20:1331-40.

6. Ko PJ, Dixon SJ. Protein palmitoylation and cancer. EMBO Rep. 2018;19:e46666.

7. Resh MD. Palmitoylation of proteins in cancer. Biochem Soc Trans. 2017;45:409-16.

8. Tabaczar S, Czogalla A, Podkalicka J, Biernatowska A, Sikorski AF. Protein palmitoylation: palmitoyltransferases and their specificity. Exp Biol Med. 2017;242:1150-7.

9. De I, Sadhukhan S. Emerging roles of DHHC-mediated protein S-palmitoylation in physiological and pathophysiological context. Eur J Cell Biol. 2018;97:319-38.

10. Yeste-Velasco M, Mao X, Grose R, Kudahetti SC, Lin D, Marzec J, et al. Identification of ZDHHC14 as a novel human tumour suppressor gene. J Pathol. 2014;232:566-77.

11. Runkle Kristin B, Kharbanda A, Stypulkowski E, Cao X-J, Wang W, Garcia Benjamin $A$, et al. Inhibition of DHHC20-mediated EGFR palmitoylation creates a dependence on EGFR signaling. Mol Cell. 2016;62:385-96.

12. Yao H, Lan J, Li C, Shi H, Brosseau JP, Wang H, et al. Inhibiting PD-L1 palmitoylation enhances T-cell immune responses against tumours. Nat Biomed Eng. 2019;3:306-17.

13. Le $X, M u J$, Peng $W$, Tang J, Xiang $Q$, Tian $S$, et al. DNA methylation downregulated ZDHHC1 suppresses tumor growth by altering cellular metabolism and inducing oxidative/ER stress-mediated apoptosis and pyroptosis. Theranostics. 2020;10:9495-511.

14. Gluck S, Ross JS, Royce M, McKenna EF Jr, Perou CM, Avisar E, et al. TP53 genomics predict higher clinical and pathologic tumor response in operable early-stage breast cancer treated with docetaxel-capecitabine $+/$ - trastuzumab. Breast Cancer Res Treat. 2012;132:781-91.

15. Cao N, Li JK, Rao YQ, Liu H, Wu J, Li B, et al. A potential role for protein palmitoylation and zDHHC16 in DNA damage response. BMC Mol Biol. 2016;17:12.

16. Drisdel RC, Green WN. Labeling and quantifying sites of protein palmitoylation. Biotechniques. 2004;36:276-85.

17. Rainwater R, Parks D, Anderson ME, Tegtmeyer P, Mann K. Role of cysteine residues in regulation of p53 function. Mol Cell Biol. 1995;15:3892-903.

18. Levine AJ. p53: 800 million years of evolution and 40 years of discovery. Nat Rev Cancer. 2020;20:471-80.

19. Dai C, Gu W. p53 post-translational modification: deregulated in tumorigenesis. Trends Mol Med. 2010;16:528-36.

20. Ashcroft M, Kubbutat MHG, Vousden KH. Regulation of p53 function and stability by phosphorylation. Mol Cell Biol. 1999;19:1751-8.

21. Chamberlain LH, Shipston MJ. The physiology of protein S-acylation. Physiol Rev. 2015;95:341-76.

22. Blanc M, David F, Abrami L, Migliozzi D, Armand F, Burgi J, et al. SwissPalm: protein palmitoylation database. F1000Research. 2015;4:261.

23. Leong WF, Zhou T, Lim GL, Li B. Protein palmitoylation regulates osteoblast differentiation through BMP-induced osterix expression. PLoS ONE. 2009;4:e4135.
24. Daniotti JL, Pedro MP, Valdez, Taubas J. The role of S-acylation in protein trafficking. Traffic. 2017;18:699-710.

25. Brownlee C, Heald R. Importin alpha partitioning to the plasma membrane regulates intracellular scaling. Cell. 2019;176:805-15 e8.

26. Goldfarb DS, Corbett AH, Mason DA, Harreman MT, Adam SA. Importin alpha: a multipurpose nuclear-transport receptor. Trends Cell Biol. 2004;14:505-14.

27. Jung $\mathrm{H}$, Seong $\mathrm{HA}, \mathrm{Ha} H$. NM23-H1 tumor suppressor and its interacting partner STRAP activate p53 function. J Biol Chem. 2007;282:35293-307.

28. Parks $D$, Bolinger $R$, Mann K. Redox state regulates binding of $p 53$ to sequencespecific DNA, but not to non-specific or mismatched DNA. Nucleic acids Res. 1997;25:1289-95.

29. HG G, JK C, J S, B S, C R, WZ W, et al. Distribution of p53 and K-ras mutations in human lung cancer tissues. Carcinogenesis. 1997;18:473-8.

30. Barretina J, Caponigro G, Stransky N, Venkatesan K, Margolin AA, Kim S, et al. The cancer cell line Encyclopedia enables predictive modelling of anticancer drug sensitivity. Nature.2012;483:603-7.

31. Fraser NJ, Howie J, Wypijewski KJ, Fuller W. Therapeutic targeting of protein Sacylation for the treatment of disease. Biochem Soc Trans. 2020;48:281-90.

32. Tao Q, Huang H, Geiman T, Lim C, Fu L, Qiu G, et al. Defective de novo methylation of viral and cellular DNA sequences in ICF syndrome cells. Hum Mol Genet. 2002;11:2091-102.

33. Zhang Y, Fan J, Fan Y, Li L, He X, Xiang Q, et al. The new $6 q 27$ tumor suppressor DACT2, frequently silenced by CpG methylation, sensitizes nasopharyngeal cancer cells to paclitaxel and 5-FU toxicity via beta-catenin/Cdc25c signaling and G2/M arrest. Clin Epigenetics. 2018;10:26.

34. Xiang T, Tang J, Li L, Peng W, Du Z, Wang X, et al. Tumor suppressive BTB/POZ zinc-finger protein ZBTB28 inhibits oncogenic BCL6/ZBTB27 signaling to maintain p53 transcription in multiple carcinogenesis. Theranostics.2019;9:8182-95.

35. Brigidi GS, Bamji SX. Detection of protein palmitoylation in cultured hippocampal neurons by immunoprecipitation and acyl-biotin exchange (ABE). J vis exp: JoVE. 2013;72:50031.

\section{ACKNOWLEDGEMENTS}

This study was supported by the National Natural Science Foundation of China (\#81872380, \#81572769), Natural Science Foundation of Chongqing (cstc2018jscxmszdX0039), and Research Grants Council, University Grants Committee (HK) (\#14115920).

\section{COMPETING INTERESTS}

The authors declare no competing interests.

\section{ADDITIONAL INFORMATION}

Supplementary information The online version contains supplementary material available at https://doi.org/10.1038/s41388-021-01949-5.

Correspondence and requests for materials should be addressed to T.X.

Reprints and permission information is available at http://www.nature.com/ reprints

Publisher's note Springer Nature remains neutral with regard to jurisdictional claims in published maps and institutional affiliations.

Open Access This article is licensed under a Creative Commons Attribution 4.0 International License, which permits use, sharing, adaptation, distribution and reproduction in any medium or format, as long as you give appropriate credit to the original author(s) and the source, provide a link to the Creative Commons license, and indicate if changes were made. The images or other third party material in this article are included in the article's Creative Commons license, unless indicated otherwise in a credit line to the material. If material is not included in the article's Creative Commons license and your intended use is not permitted by statutory regulation or exceeds the permitted use, you will need to obtain permission directly from the copyright holder. To view a copy of this license, visit http://creativecommons. org/licenses/by/4.0/.

(c) The Author(s) 2021 